\title{
Antimicrobial activity of mul-1867, a novel antimicrobial compound, against multidrug-resistant Pseudomonas aeruginosa
}

\author{
George Tetz $^{1 *}$, Daria Vikina ${ }^{2}$ and Victor Tetz ${ }^{2}$
}

\begin{abstract}
Background: There is an urgent need for new antimicrobial compounds to treat various lung infections caused by multidrug-resistant Pseudomonas aeruginosa (MDR-PA).

Methods: We studied the potency of Mul-1867 against MDR-PA isolates from patients with cystic fibrosis, chronic obstructive pulmonary disease, and ventilator-associated pneumonia. The minimal inhibitory concentrations (MICs) and minimum biofilm eliminating concentrations (MBECs), defined as the concentrations of drug that kill $50 \%$ $\left(\mathrm{MBEC}_{50}\right), 90 \%\left(\mathrm{MBEC}_{90}\right)$, and $100 \%\left(\mathrm{MBEC}_{100}\right)$ of the bacteria in preformed biofilms, were determined by using the broth macrodilution method.

Results: Mul-1867 exhibited significant activity against MDR-PA and susceptible control strains, with MICs ranging from 1.0 to $8.0 \mu \mathrm{g} / \mathrm{mL}$. Mul-1867 also possesses anti-biofilm activity against mucoid and non-mucoid 24-h-old MDRPA biofilms. The MBEC ${ }_{50}$ value was equal to onefold the MIC. The MBEC 90 value ranged from two to fourfold the MIC. Moreover, Mul-1867 completely eradicated mature biofilms at the concentrations tested, with MBEC $_{100}$ values ranging between 16- and 32-fold the MIC. Mul-1867 was non-toxic to Madin-Darby canine kidney (MDCK) cells at concentrations up to $256 \mu \mathrm{g} / \mathrm{mL}$.
\end{abstract}

Conclusion: Overall, these data indicate that Mul-1867 is a promising locally acting antimicrobial for the treatment and prevention of P. aeruginosa infections.

Keywords: Biofilms, Mucoid, Non-mucoid, Multidrug-resistant, P. aeruginosa, Cystic fibrosis

\section{Background}

Pseudomonas aeruginosa is an important pathogen contributing to lung infections in patients with pulmonary diseases such as cystic fibrosis (CF), chronic obstructive pulmonary disease (COPD), and ventilator-associated pneumonia (VAP) [1-3]. By 21 years of age, most CF patients are chronically infected with $P$. aeruginosa that, once established, is difficult to eradicate and is associated with major morbidity [4-6]. P. aeruginosa is also an important contributor to acute exacerbations in COPD

\footnotetext{
*Correspondence: georgetets@gmail.com

${ }^{1}$ TGV-Laboratories LLC, 303 5th avenue, \# 2012, New York, NY 10016, USA Full list of author information is available at the end of the article
}

and VAP [7]. Some P. aeruginosa strains are mucoid due to overexpression of alginate, and are less susceptible to the host immune system [8]. Overproduction of alginate, and conversion of $P$. aeruginosa to a mucoid phenotype, are considered markers of chronic lung infections and indicate a decreased life expectancy and a more rapid decline in pulmonary function [9].

Both mucoid and non-mucoid strains of $P$. aeruginosa form biofilms that are associated with persistence in chronic infections, and also contribute to bacterial colonization in acute infections [10]. Established biofilms display high resistance to antimicrobials, and finding ways to disrupt these films is a critical goal in combatting these infections $[11,12]$. P. aeruginosa survival in 
biofilms may be determined by multiple factors including the surface film and the presence of extracellular polymeric substances $[13,14]$. Moreover, clinical isolates of multidrug resistant $P$. aeruginosa (MDR-PA) are being observed with increasing frequency [15]. Thus, biofilms formed by MDR-PA are a serious and often fatal problem for patients with lung infections, and many antibiotics are ineffective against bacteria in biofilm communities at concentrations of 10-1000 times the expected minimum inhibitory concentration (MIC) [16].

During the last decade, it became evident that existing antibiotics were unable to treat respiratory infections caused by $P$. aeruginosa effectively [17]. Lung infections caused by MDR-PA were poorly responsive even to nebulized therapy when the aerosolized antibiotics achieved high concentrations directly at the pathological site [18]. It was also shown that many antibiotics, even when able to decrease the number of live bacteria within the biofilm, could not completely eradicate preformed biofilms [19].

The poor therapeutic results with existing antibiotics revealed a critical need for the development of new antimicrobials active against MDR-PA that can be used to treat lung infections. The goal of this study was to examine in vitro MICs and minimal biofilm eradication concentrations (MBECs) of the novel antimicrobial compound, Mul-1867 (poly-N,N'-hexamethyleneguanidine-poly-N1,N4-hexamethyleneaminoguanidine; Fig. 1). The mechanism of action of Mul-1867 involves a nonspecific attack on the cell walls of MDR-PA isolated from patients with respiratory tract infections [20]. Furthermore, the anti-biofilm efficacy of Mul-1867 against preformed mucoid and non-mucoid 24-h-old biofilms of MDR-PA was assessed.

\section{Methods}

\section{Test substance and antibiotics}

Mul-1867 (Fig. 1) was synthesized by TGV-Therapeutics (New York, NY, USA).

The antibiotics used for these studies included amikacin, aztreonam, ceftazidime, meropenem, piperacillin, and tobramycin, which are currently administered to treat lung infections in patients with CF, COPD, or VAP. These antibiotics were purchased from Sigma-Aldrich (St. Louis, MO, USA).

\section{Bacterial strains and growth conditions}

MDR-PA isolates from CF patients including the AGR14, MR45, and VT-CF-234 strains, as well as one susceptible isolate, SEV 7; were obtained from the CF Foundation Therapeutics Development Network Resource Center for Microbiology at Seattle Children's Hospital (Seattle, WA, USA) and from the Institute of Human Microbiology, LLC (DE, USA). MDR-PA isolates from COPD and VAP patients, VT-COPD-274 and VT-VAP-72, respectively, were supplied by the Institute of Human Microbiology. The three strains classified phenotypically as mucoid were SEV 7, VT-CF-234, and VT-COPD-274. The strain used for quality control of the antibiotic panels was $P$. aeruginosa ATCC 27853 from the American Type Culture Collection (Manassas, VA, USA).

All bacterial strains were subcultured from freezer stocks onto Mueller-Hinton agar plates (Oxoid Ltd., London, England) and incubated at $37{ }^{\circ} \mathrm{C}$ overnight. All subsequent liquid subcultures were derived from colonies isolated from these plates and were grown in MuellerHinton broth (MHB) (Oxoid). The isolates were categorized as susceptible, intermediate, or resistant according to the Clinical and Laboratory Standards Institute (CLSI) breakpoints guidelines (Table 1) [21].

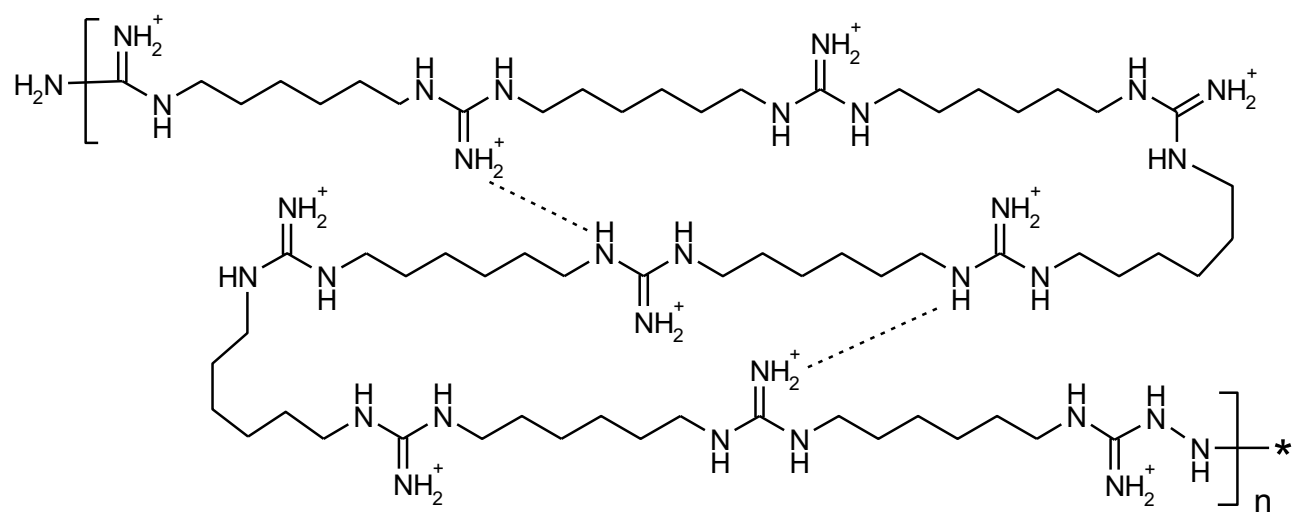

Fig. 1 Chemical structure of Mul-1867n $=1-20$ 
Table 1 Bactericidal activities of Mul-1867 and other antibacterials against non-mucoid $P$. aeruginosa isolates

\begin{tabular}{|c|c|c|c|c|c|}
\hline \multirow[t]{2}{*}{ Antibacterials } & \multirow{2}{*}{$\begin{array}{l}\text { Susceptibility } \\
\text { breakpoint }(\mu \mathrm{g} / \mathrm{mL})\end{array}$} & \multicolumn{4}{|c|}{ P. aeruginosa strain minimal inhibitory concentration $(\mu \mathrm{g} / \mathrm{mL})$} \\
\hline & & AGR14 & MR 45 & VT-VAP-72 & ATCC 27853 \\
\hline Mul-1867 & $N D^{a}$ & 8 & 8 & 4 & 1 \\
\hline Amikacin & $\leq 16$ & 128 & 256 & 128 & 4 \\
\hline Aztreonam & $\leq 8$ & 32 & 128 & 64 & 2 \\
\hline Ceftazidime & $\leq 8$ & 32 & 32 & 16 & 2 \\
\hline Meropenem & $\leq 2$ & 32 & 64 & 32 & 1 \\
\hline Piperacillin & $\leq 16$ & $>256$ & 256 & 128 & 4 \\
\hline Tobramycin & $\leq 4$ & 32 & 32 & 32 & 1 \\
\hline
\end{tabular}

a $N D$ no data

\section{In vitro antimicrobial susceptibility testing}

The MICs for antimicrobials were determined by the broth macrodilution method according to CLSI guideline $[22,23]$. A standard bacterial inoculum of $5 \times 10^{5}$ colony forming units (CFU)/mL was used. Serial twofold dilutions of the antimicrobials were prepared in cationadjusted MHB. The MIC was defined as the lowest concentration of antibiotic that completely inhibited visible growth. Experiments were conducted in triplicate.

\section{Effect of Mul-1867 against $P$. aeruginosa biofilms}

In each well of a 96-well flat-bottom polystyrene tissue culture microtiter plate (Sarstedt, Numbrecht, Germany), $200 \mu \mathrm{L}$ of a standardized $P$. aeruginosa inoculum $\left(5 \times 10^{5}\right.$ $\mathrm{CFU} / \mathrm{mL}$ ) in cation-adjusted MHB were added. Following a $24 \mathrm{~h}$ incubation period at $37{ }^{\circ} \mathrm{C}$, biofilm samples were washed twice with phosphate-buffered saline to remove non-adherent bacteria and then exposed for $24 \mathrm{~h}$ to $200 \mu \mathrm{L}$ of MHB containing Mul-1867 at 1-, 2-, 4-, 8-, 16-, 32-, and 64-fold the MIC. Untreated biofilms were used as the negative controls. After the exposure, well contents were aspirated to prevent antimicrobial carryover and each well was washed three times with sterile deionized water.

To estimate the CFU number, biofilms were scraped thoroughly, with particular attention paid to the well edges [11]. The well contents were aspirated, placed in $1 \mathrm{~mL}$ of isotonic phosphate buffer $(0.15 \mathrm{M}, \mathrm{pH} 7.2)$, and the total CFU number was determined by serial dilution and plating on Mueller-Hinton agar.

The MBECs were determined as the concentrations of drug that killed $50 \%\left(\mathrm{MBEC}_{50}\right), 90 \%\left(\mathrm{MBEC}_{90}\right)$, and $100 \%\left(\mathrm{MBEC}_{100}\right)$ of the bacteria in preformed biofilms compared to the untreated control (100\% viability). MBEC sensitivities were determined using the 2012 Clinical and Laboratory Standards Institute guidelines for interpretation [23]. Tests were performed in triplicate on three separate occasions, and the results were averaged.

\section{Cytotoxicity assay}

Cytotoxicity of Mul-1867 on Madin-Darby canine kidney (MDCK) cells was determined by the microculture tetrazolium (MTT) assay. Briefly, cells $\left(1 \times 10^{5} /\right.$ well $)$ were plated in $100 \mu \mathrm{L}$ of minimal essential media in 96-well plates and incubated overnight in a $5 \% \mathrm{CO}_{2}$ atmosphere. Serial dilutions of Mul-1867, with final concentrations corresponding to a range of $8-256 \mu \mathrm{g} / \mathrm{mL}$, were added to the cells that were then incubated at $37{ }^{\circ} \mathrm{C}$ for a further $24 \mathrm{~h}$. Control wells were treated with sterile water. MTT analysis was then performed based on the standard method [24].

After incubation, an aliquot of [3-(4,5-Dimethylthiazol2-yl)-2,5-diphenyltetrazolium bromide] solution (SigmaAldrich, St. Louis, MO, USA) at $0.5 \mathrm{mg} / \mathrm{mL}$ was added to each well, and following a further $30 \mathrm{~min}$ incubation at $37^{\circ} \mathrm{C}$, the medium was discarded and the formazan blue formed in the cells was dissolved with $100 \mu \mathrm{L}$ of dimethyl sulfoxide. Absorbance at $570 \mathrm{~nm}$ was determined with a microplate reader (Stat-Fax-2100, Awareness Technology Inc, USA). The absorbance of the formazan formed in non-treated cells was considered $100 \%$ viability.

\section{Statistical analyses}

Results of the cytotoxicity assay are presented as mean \pm standard deviation of three independent experiments. All statistical analyses were performed using the statistics package Statistica for Windows (version 5.0). Results are reported as the mean \pm standard error for each group. Non-parametric paired Wilcoxon signed rank test was applied to analyse pre- and post-challenge differences. $\mathrm{P}<0.05$ was considered significant.

\section{Results}

In vitro antimicrobial susceptibility testing of $P$. aeruginosa isolates

The MICs of Mul-1867 and other antibiotics against nonmucoid $P$. aeruginosa isolates are shown in Table 1 . 
Mul-1867 exhibited significant activity against all isolates that were resistant to amikacin, aztreonam, ceftazidime, meropenem, piperacillin, and tobramycin according to CLSI criteria, as well as against susceptible and control strains.

Of the seven MDR-PA isolates, three had the mucoid phenotype that is widespread among CF patients and is associated with reduced success of treatment. Mul-1867 exhibited a high level of antimicrobial activity against mucoid MDR-PA strains irrespective of their resistance to other antibiotics (Table 2) $[25,26]$.

There was no statistically significant difference between the activity of Mul-1867 against the mucoid vs. nonmucoid $P$. aeruginosa strains. The MIC values ranged from 1 to $8 \mu \mathrm{g} / \mathrm{mL}$ for the non-mucoid strains, and from 2 to $4 \mu \mathrm{g} / \mathrm{mL}$ for the mucoid strains.

\section{Effect of Mul-1867 against preformed P. aeruginosa biofilms}

The anti-biofilm activity of Mul-1867 against 24-h-old $P$. aeruginosa biofilms was analyzed to reveal possible differences in susceptibility patterns between non-mucoid and mucoid $P$. aeruginosa strains. The activity of Mul1867 on mature biofilms, tested at concentrations equal to a multiple of the MIC, is summarized in Table 3. After $24 \mathrm{~h}$ of growth, the CFUs of $P$. aeruginosa AGR14 and $P$. aeruginosa VT-CF-234 biofilms were $1.8 \times 10^{8} \mathrm{CFU} / \mathrm{mL}$ and $3.5 \times 10^{8} \mathrm{CFU} / \mathrm{mL}$, respectively.

A comparison between the MIC and MBEC values indicated that Mul-1867 was a highly active antimicrobial exhibiting dose-dependent effects against the $P$. aeruginosa strains tested. Mul-1867 showed MBEC $_{50}$ and $\mathrm{MBEC}_{90}$ values of 1- and 4-fold the MIC, respectively, against a non-mucoid strain. Mul-1867 displayed comparable activity against a mucoid strain with

Table 2 Minimal inhibitory concentrations of Mul-1867 and other antibacterials against mucoid $P$. aeruginosa isolates

\begin{tabular}{llrcc}
\hline Antibacterials & $\begin{array}{l}\text { Susceptibility } \\
\text { breakpoint } \\
(\boldsymbol{\mu g} / \mathbf{m L})\end{array}$ & \multicolumn{2}{l}{$\begin{array}{l}\text { P. aeruginosa strain minimal } \\
\text { inhibitory concentration }(\boldsymbol{\mu g} / \mathbf{m l})\end{array}$} \\
\cline { 3 - 5 } & SEV 7 & VT-CF-234 & VT-COPD-274 \\
\hline Mul-1867 & $\mathrm{ND}^{\mathrm{a}}$ & 2 & 2 & 4 \\
Amikacin & $\leq 16$ & 128 & 64 & 256 \\
Aztreonam & $\leq 8$ & 64 & 64 & 128 \\
Ceftazidime & $\leq 8$ & 2 & 32 & 32 \\
Piperacillin & $\leq 16$ & 2 & 64 & 128 \\
Meropenem & $\leq 2$ & 32 & 8 & 16 \\
Tobramycin & $\leq 4$ & 16 & 128 & 64 \\
\hline
\end{tabular}

a ND No data
Table 3 Susceptibility results of Mul-1867 against $P$. aeruginosa strains

\begin{tabular}{lllll}
\hline Isolate & Mucoid & $\begin{array}{l}\mathrm{MBEC}_{\mathbf{5 0}} \\
(\boldsymbol{\mu g} / \mathbf{m L})\end{array}$ & $\begin{array}{l}\mathrm{MBEC}_{\mathbf{9 0}} \\
(\boldsymbol{\mu g} / \mathbf{m L})\end{array}$ & $\begin{array}{l}\mathrm{MBEC}_{\mathbf{1 0 0}} \\
(\boldsymbol{\mu g} / \mathbf{m L})\end{array}$ \\
\hline $\begin{array}{c}\text { P. aeruginosa } \\
\text { AGR14 }\end{array}$ & No & 8 & 32 & 256 \\
$\begin{array}{c}\text { P. aeruginosa } \\
\text { VT-CF-234 }\end{array}$ & Yes & 2 & 4 & 32 \\
\hline
\end{tabular}

MBEC minimum biofilm eliminating concentrations

$\mathrm{MBEC}_{50}$ and $\mathrm{MBEC}_{90}$ values of 1 - and twofold the MIC, respectively.

Reduction in cell numbers by $90 \%$ suggested that Mul1867 has the potential for biofilm eradication, which is an important goal in antimicrobial treatment. Thus, we determined the $\mathrm{MBEC}_{100}$ after treatment of P. aeruginosa biofilms with Mul-1867 for $24 \mathrm{~h}$.

In this assay, Mul-1867 completely eradicated mature $P$. aeruginosa biofilms at the concentrations tested, with $\mathrm{MBEC}_{100}$ values of 32 - and 16 -fold the MIC for the AGR14 and VT-CF-234 strains, respectively. The ability to eradicate a biofilm at a concentration eightfold higher than that required to reduce biofilm bacteria by $90 \%$ suggested that Mul-1867 possesses significant anti-biofilm activity.

\section{Cell cytotoxicity of Mul-1867}

The compound did not exhibit any statistically significant cytotoxicity against MDCK cells at concentrations ranging from 8 to $256 \mu \mathrm{g} / \mathrm{mL}$, as tested by the MTT assay $24 \mathrm{~h}$ after challenging with Mul-1867 (Fig. 2).

\section{Discussion}

P. aeruginosa presents substantial problems for patients with CF, and is a major predictor of morbidity [27]. For the last decade, the emergence of MDR-PA strains has posed treatment challenges in patients with COPD and VAP [28]. In the current study, we found that a novel

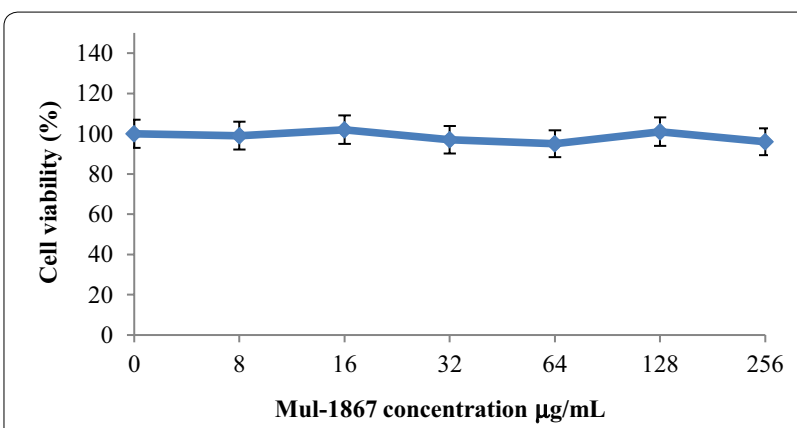

Fig. 2 Cytotoxic effect of Mul-1867 on Madin-Darby canine kidney cell lines as determined by the microculture tetrazolium assay 
drug candidate, Mul-1867, exhibits a high level of antimicrobial activity against both MDR and susceptible clinical isolates of $P$. aeruginosa from patients with life-threatening lung infections.

We showed previously that Mul-1867 is a fast-acting antimicrobial whose mechanism of action involves a nonspecific attack on the bacterial cell wall that causes leakage of cell contents [20]. Mucoid strains lead to the persistence of $P$. aeruginosa infections. The data available in the literature concerning the effect of mucoidity on antimicrobial susceptibility show some differences depending on the type of antibiotic being tested [29]. Our data indicate that there is no cross-resistance between amikacin, aztreonam, ceftazidime, meropenem, piperacillin, and tobramycin vs. Mul-1867 against susceptible and MDR-PA strains tested in this study.

$P$. aeruginosa biofilms respond poorly to treatment with existing medicines due to the presence of additional barriers including a surface film or extracellular polymeric substances that reduce antibiotic penetration [30]. Biofilms formed by MDR-PA are almost completely insensitive to treatment with antibiotics that have high MBEC values that cannot be achieved at the site of infection using currently recommended dosages, or even with local administration directly to the site of infection [31]. There are also reports that biofilms formed by some strains of $P$. aeruginosa cannot be eradicated at any concentration of antibiotics that are commonly used for therapy. Importantly, however, Mul-1867 was equally effective against mucoid and non-mucoid $P$. aeruginosa strains.

The $\mathrm{MBEC}_{90} / \mathrm{MIC}$ ratio is an important parameter for choosing the best antibacterial to treat $P$. aeruginosa biofilm-associated infections (lower is better). Our studies revealed that Mul-1867 possessed low $\mathrm{MBEC}_{90} / \mathrm{MIC}$ ratios (between two and four). Moreover, biofilms formed by both non-mucoid and mucoid $P$. aeruginosa strains were totally eradicated with Mul-1867 at concentrations of 32- and 16-fold the MIC, respectively.

Positively charged antibiotics bind to extracellular polymeric substance and extracellular DNA, which decreases their bioavailability [30]. Mul-1867 is a positively charged molecule, and its antimicrobial activity was also reduced by the biofilm's components. However, its activity level remained high enough to achieve total eradication of biofilms.

Cell viability assessed by the MTT assay revealed that Mul-1867 did not display cytotoxic effects at the tested concentrations (between 8 to $256 \mu \mathrm{g} / \mathrm{mL}$ ). The absence of apparent toxicity to MDCK cells at the highest concentration of Mul-1867 that was used against the least sensitive $P$. aeruginosa strain tested in this study (AGR14) is a notable attribute of this topical antimicrobial compound.
The high levels of Mul-1867 activity against the clinical isolates tested in this study are consistent with those found in our previous work [20]. This demonstrates the broad antimicrobial activity of this molecule against planktonic and sessile gram-positive and gram-negative bacteria. The efficacy of Mul-1867 against both nonmucoid and mucoid strains of $P$. aeruginosa raises the possibility that it may serve as a locally acting antimicrobial compound and may be able to successfully eradicate biofilm infections caused by $P$. aeruginosa.

\section{Abbreviations}

CF: cystic fibrosis; CFU: colony forming units; CLSI: Clinical and Laboratory Standards Institute; COPD: chronic obstructive pulmonary disease; MBEC: minimum biofilm eliminating concentrations; MDCK: madin-Darby canine kidney; MDR-PA: multidrug-resistant Pseudomonas aeruginosa; MHB: Mueller-Hinton broth; MIC: minimal inhibitory concentration; VAP: ventilator-associated pneumonia.

\section{Authors' contributions}

Conceived and designed the experiments VT GT. Performed the experiments: VT DV GT. Analyzed the data: VT GT. Contributed reagents/materials/analysis tools: VT DV GT. Contributed to the writing of the manuscript: GT. All authors read and approved the final manuscript.

\section{Author details}

${ }^{1}$ TGV-Laboratories LLC, 303 5th avenue, \# 2012, New York, NY 10016, USA.

${ }^{2}$ Institute of Human Microbiology, New York, NY 10016, USA.

\section{Acknowledgements}

The authors thank Jane Burns and Adam Griffith from Seattle Children's Research Institute for generously supplying the clinical isolates.

\section{Competing interests}

The authors declare that they have no competing interests.

Received: 10 December 2015 Accepted: 14 March 2016

Published online: 22 March 2016

\section{References}

1. Garcia-Vidal C, Almagro P, Romaní V, Rodríguez-Carballeira M, Cuchi E, Canales L, Garau J, et al. Pseudomonas aeruginosa in patients hospitalised for COPD exacerbation: a prospective study. Eur Respir J. 2009;34:1072-8.

2. Folkesson A, Jelsbak L, Yang L, Johansen HK, Ciofu O, Hoiby N, et al. Adaptation of Pseudomonas aeruginosa to the cystic fibrosis airway: an evolutionary perspective. Nat Rev Microbiol. 2012;10:841-51.

3. Peña C, Gómez-Zorrilla S, Oriol I, Tubau F, Dominguez MA, Pujol M, et al. Impact of multidrug resistance on Pseudomonas aeruginosa ventilatorassociated pneumonia outcome: predictors of early and crude mortality. Eur J Clin Microbiol Infect Dis. 2013;32:413-20.

4. Saiman L, Marshall BC, Mayer-Hamblett N, Burns JL, Quittner AL, Cibene $D A$, et al. Azithromycin in patients with cystic fibrosis chronically infected with Pseudomonas aeruginosa: a randomized controlled trial. JAMA. 2003;290:1749-56.

5. Mowat E, Paterson S, Fothergill JL, Wright EA, Ledson MJ, Walshaw MJ, et al. Pseudomonas aeruginosa population diversity and turnover in cystic fibrosis chronic infections. Am J Respir Crit Care Med. 2011;183:1674-9.

6. Schelstraete P, Haerynck F, Van daele S, Deseyne S, De Baets F. Eradication therapy for Pseudomonas aeruginosa colonization episodes in cystic fibrosis patients not chronically colonized by P. aeruginosa. J Cyst Fibros. 2013;12:1-8. 
7. Domenech A, Puig C, Martí S, Santos S, Fernández A, Calatayud L, et al. Infectious etiology of acute exacerbations in severe COPD patients. J Infect. 2013;67:516-23.

8. Owlia P, Nosrati R, Alaghehbandan R, Lari AR. Antimicrobial susceptibility differences among mucoid and non-mucoid Pseudomonas aeruginosa isolates. GMS Hyg Infect Control. 2014;9:13.

9. Govan JR, Deretic V. Microbial pathogenesis in cystic fibrosis: mucoid Pseudomonas aeruginosa and Burkholderia cepacia. Microbiol Rev. 1996;60:539-74.

10. Costerton JW, Stewart PS, Greenberg EP. Bacterial biofilms: a common cause of persistent infections. Science. 1999;284:1318-22.

11. Tetz GV, Artemenko NK, Tetz WV. Effect of DNase and antibiotics on biofilm characteristics. Antimicrob Agents Chemother. 2009:53:1204-9.

12. Lopes SP, Ceri H, Azevedo NF, Pereira MO. Antibiotic resistance of mixed biofilms in cystic fibrosis: impact of emerging microorganisms on treatment of infection. Int J Antimicrob Agents. 2012;40:260-3.

13. Tetz W, Korobov VP, Artemenko NK, Lemkina LM, Panjkova NV, Tetz GV. Extracellular phospholipids of isolated bacterial communities. Biofilms 2004;1:149-55.

14. Hall-Stoodley L, Costerton JW, Stoodley P. Bacterial biofilms: from the natural environment to infectious diseases. Nat Rev Microbiol. 2004:2:95-108.

15. Breidenstein EB, de la Fuente-Núñez C, Hancock RE. Pseudomonas aeruginosa: all roads lead to resistance. Trends Microbiol. 2011;19:419-26.

16. Rodríguez-Martínez JM, Pascual A. Antimicrobial resistance in bacterial biofilms. Rev Med Microbiol. 2006;17:65-75.

17. Döring G, Flume P, Heijerman H, Elborn JS. Consensus Study Group. Treatment of lung infection in patients with cystic fibrosis: current and future strategies. J Cyst Fibros. 2012;11:461-79.

18. Morosini Ml, García-Castillo M, Loza E, Pérez-Vázquez M, Baquero F, Cantón R. Breakpoints for predicting Pseudomonas aeruginosa susceptibility to inhaled tobramycin in cystic fibrosis patients: use of high-range Etest strips. J Clin Microbiol. 2005:43:4480-5.

19. Reiter KC, Sambrano GE, Villa B, Paim T, Oliveira C, d'Azevedo PA. Rifampicin fails to eradicate mature biofilm formed by methicillin-resistant Staphylococcus aureus. Rev Soc Bras Med Trop. 2012;45:471-4.

20. Tetz G, Tetz V. In vitro antimicrobial activity of a novel compound, Mul1867, against clinically important bacteria. Antimicrob Resist Infect Control. 2015;4:45

21. Clinical and Laboratory Standards Institute. Performance standards for antimicrobial susceptibility testing. Twenty-first informational supplement (M100-S21). Clinical and Laboratory Standards Institute Wayne; 2011.

22. Clinical and Laboratory Standards Institute. Methods for determining bactericidal activity of antimicrobial agents; approved guideline (M26-A) Wayne: Clinical and Laboratory Standards Institute; 1999.

23. Clinical and Laboratory Standards Institute. Methods for dilution antimicrobial susceptibility tests for bacteria that grow aerobically; approved standard, ninth edition (M07-A9). Wayne: Clinical and Laboratory Standards Institute; 2012.

24. Sieuwerts AM, Klijn JG, Peters HA, Foekens JA. The MTT tetrazolium salt assay scrutinized: how to use this assay reliably to measure metabolic activity of cell cultures in vitro for the assessment of growth characteristics, IC50-values and cell survival. Eur J Clin Chem Clin Biochem. 1995;33:813-23

25. Mayer-Hamblett N, Rosenfeld M, Gibson RL, Ramsey BW, Kulasekara HD, Retsch-Bogart GZ, et al. Pseudomonas aeruginosa in vitro phenotypes distinguish cystic fibrosis infection stages and outcomes. Am J Respir Crit Care Med. 2014;190:289-97.

26. Lam JC, Somayaji R, Surette MG, Rabin HR, Parkins MD. Reduction in Pseudomonas aeruginosa sputum density during a cystic fibrosis pulmonary exacerbation does not predict clinical response. BMC Infect Dis. 2015;15:145.

27. Flume PA, VanDevanter DR. Clinical applications of pulmonary delivery of antibiotics. Adv Drug Deliv Rev. 2015:85:1-6.

28. Fricks-Lima J, Hendrickson CM, Allgaier M, Zhuo H, Wiener-Kronish JP, Lynch SV, et al. Differences in biofilm formation and antimicrobial resistance of Pseudomonas aeruginosa isolated from airways of mechanically ventilated patients and cystic fibrosis patients. Int J Antimicrob Agents. 2011;37:309-15.

29. Schülin T. In vitro activity of the aerosolized agents colistin and tobramycin and five intravenous agents against Pseudomonas aeruginosa isolated from cystic fibrosis patients in southwestern Germany. J Antimicrob Chemother. 2002;49:403-6.

30. Messiaen AS, Nelis H, Coenye T. Investigating the role of matrix components in protection of Burkholderia cepacia complex biofilms against tobramycin. J Cyst Fibros. 2014;13:56-62.

31. Hengzhuang W, Ciofu O, Yang L, Wu H, Song Z, Oliver A, et al. High $\beta$-lactamase levels change the pharmacodynamics of $\beta$-lactam antibiotics in Pseudomonas aeruginosa biofilms. Antimicrob Agents Chemother. 2013:57:196-204.

\section{Submit your next manuscript to BioMed Central and we will help you at every step:}

- We accept pre-submission inquiries

- Our selector tool helps you to find the most relevant journal

- We provide round the clock customer support

- Convenient online submission

- Thorough peer review

- Inclusion in PubMed and all major indexing services

- Maximum visibility for your research

Submit your manuscript at www.biomedcentral.com/submit
( Biomed Central 